\title{
Sirtuin-1 in Egyptian patients with coronary artery disease
}

\author{
AL-Shabrawy M. Abdelnabi ${ }^{1}$, Imam M. Esmayel ${ }^{1}$, Samia Hussein ${ }^{2 *}$ (D, Reham M. Ali ${ }^{1}$ and \\ Alhoussein Alsayed AbdelAal ${ }^{1}$
}

\begin{abstract}
Background: Coronary artery disease (CAD) represents the leading cause of death worldwide. Animal and human studies have demonstrated that silent information regulator 1 (SIRT1) is involved in a wide range of physiological and pathological processes. This study aimed to measure the plasma level of SIRT1 in patients with CAD and explore its correlation with cardiovascular risk factors.
\end{abstract}

Results: Plasma SIRT1 was significantly lower in patients with chronic coronary syndrome (CCS) than in those in the control group and was significantly lower in patients with both acute myocardial infarction and unstable angina than in those in the control group and with CCS. Moreover, plasma SIRT1 was positively correlated with platelet count and negatively correlated with cholesterol and triglyceride levels.

Conclusions: The plasma level of SIRT1 is lower in patients with CAD compared to control and it could be a possible marker for this disease. Multi-center studies with follow-up measurements are recommended for further investigation.

Keywords: Atherosclerosis, Coronary heart disease, Sirtuin

\section{Background}

Coronary artery disease (CAD) involves chronic coronary syndrome (CCS) and acute coronary syndrome (ACS). ACS includes unstable angina (UA) and acute myocardial infarction (AMI). Most cases of ACS arise from the disruption of a previously non-severe lesion (an atherosclerotic lesion that was previously hemodynamically insignificant yet vulnerable to rupture). The vulnerable plaque is made of a large lipid pool, numerous inflammatory cells, and a thin fibrous cap [1].

The pathogenesis of ACS has been attributed primarily to atherosclerosis. The physical crack of the atherosclerotic plaque that develops in patients with ACS represents practically almost all cases of acute coronary thrombi [2].

\footnotetext{
*Correspondence: samiahussein82@hotmail.com

${ }^{2}$ Medical Biochemistry and Molecular Biology Department, Faculty

of Medicine, Zagazig University, Zagazig, Egypt

Full list of author information is available at the end of the article
}

It was found that caloric restriction, resveratrol, and ischemic preconditioning can protect against ischemic injury to the heart [3]. The underlying mechanisms of these interventions appear to be under the control of a nicotinamide adenine dinucleotide (NAD)-dependent histone deacetylase named silent information regulator 1 (SIRT1) [4]. SIRT1 is a member of the class III group of histone deacetylases, collectively called sirtuins. The mammalian sirtuin family consists of seven members designated SIRT1 through SIRT7.

SIRT1 engages in a wide scope of physiological and pathological processes [5]. However, data on sirtuins in human cardiovascular diseases (CVD) are scant [6]. These proteins assume significant functions in guaranteeing cardiovascular homeostasis under physiological and stress conditions [7] and can influence cardiac and endothelial cells either directly or indirectly by systemic regulation [8].

A recent study found that SIRT1 inhibition causes oxidative stress and inflammation in patients with CAD, whereas its activation reverses these atherosclerotic 
events [9]. The results of this study may provide new knowledge for the management of CAD patients. However, the expression and activity of SIRT1 must be strictly controlled [10].

Several previous studies have assessed plasma SIRT1 levels in patients with CAD. However, there is still a controversy in results. Doulamis et al. [11] stated that SIRT1 has a protective role in advanced CAD and reported low values [11]. On the other side, Kizıltunç et al. [12] found that serum SIRT 1, 3, and 6 levels in AMI patients were similar to those in normal coronary patients. This study denied the possible protective effects of SIRT 1, 3, and 6 in AMI patients.

Another study reported a significant inverse correlation between circulatory SIRT1 and epicardial fat thickness, which is a useful marker of the severity of CAD [13]. Interestingly, a study reported that cardiomyocytespecific deletion of the SIRT1 gene sensitizes the myocardium to ischemia and reperfusion injury [14]. Animal and human studies have demonstrated that SIRT1 has a credible protective function in myocardial ischemic injury [15].

In our study, we aimed to measure the plasma level of SIRT1 in CAD patients, assess its potential role as a candidate biomarker for predicting the risk of $\mathrm{CAD}$, and explore the correlation between SIRT1 and cardiovascular risk factors.

\section{Methods}

This is a case-control study that was carried out at the Internal Medicine and Medical Biochemistry and Molecular Biology Departments. It included 80 individuals divided into the following two groups: Group I (controls), 40 normal individuals; Group II (40 cases), subdivided into Group IIa (CAD with CCS [12 individuals]), Group IIb (CAD with unstable angina (UA) [14 individuals]), and Group IIc (AMI [14 individuals]).

Written informed consent was obtained from the patients or their relatives with an explanation of the study procedure and possible associated hazards. The study was approved by the Institute Review Board.

We included patients with spontaneous ACS or CCS aged between 40 and 60 years admitted through the outpatient and emergency departments. We excluded patients with ACS that had undergone a previous intervention (percutaneous coronary intervention or coronary artery bypass grafting), and patients with any of the following: significant valvular heart disease, hematological disease, malignancy, liver or renal disease, systemic inflammatory disease, active infection, autoimmune disease, and cardiogenic shock. We also excluded patients on steroids (whatever the indication), patients with high total cholesterol levels (above $200 \mathrm{mg} / \mathrm{dL}$ ), current and former smokers, and obese individuals (body mass index of 30 or higher).

All individuals were subjected to full history taking and physical examination. Peripheral venous blood samples were collected within $24 \mathrm{~h}$ of admission for complete blood count $(\mathrm{CBC})$, random blood glucose (RBG), renal function testing, creatine kinase-MB (CK-MB), lipid profile, and SIRT1 measurements. CBC testing was performed using an automated hematology analyzer (BeneSphera, the Netherlands). Serum creatinine and urea, lipid profiles, CK-MB and RBG were measured using a spectrophotometer (Sunostik, Changchun, China). The reagents were provided by (Spinreact, Spain). The plasma SIRT1 level was measured using an enzyme-linked immunosorbent assay (Wuhan Boster Biological Technology Ltd, Wuhan, People's Republic of China).

\subsection{Statistical analysis}

All data were collected, tabulated, and statistically analyzed using SPSS 19.0 for Windows (SPSS Inc., Chicago, IL, USA) and MedCalc 13 for Windows (MedCalc Software BVBA, Ostend, Belgium). Continuous data were presented as the mean \pm standard deviation (SD) and categorical data were presented as frequencies and percentages. We used the Shapiro-Wilk test to check continuous variables for normality. The independent Student $t$ test was used to compare two groups of normally distributed data. To compare two groups of non-normally distributed data, we used the Mann-Whitney $U$ test, and to compare more than two groups of non-normally distributed data, we used the Kruskal-Wallis test. Percentages of categorical variables were compared using the Chi-square $\left(x^{2}\right)$ test. Correlations between variables were assessed using the Pearson correlation coefficient. A $p<0.05$ was considered statistically significant and $p<0.001$ was considered highly statistically significant.

\section{Results}

There was no significant difference in age, sex, hemoglobin, RBG, or serum creatinine levels between the study groups $(p=0.081,0.45,0.74,0.477$ and 0.054 respectively). WBCs, platelet count, urea, and BUN differed significantly between the study groups $(p<0.001$ for each). In the AMI group, platelet count, WBC count, urea, BUN, and CK-MB were significantly higher and platelet count was significantly lower than in the other groups $(p<0.05)$. (Table 1$)$.

Regarding the lipid profile, total cholesterol, TG, and LDL were significantly lower and HDL was significantly higher in the control group than in the other groups $(p<0.001)$ (Table 1$)$.

There was a significant difference in CK-MB levels between the study groups $(p<0.001)$, and it was 
Table 1 Demographic and biochemical data of the studied groups

\begin{tabular}{|c|c|c|c|c|c|}
\hline & Control & CCS & Unstable angina & AMI & $p$ \\
\hline Age (years) & $51.45 \pm 5.32$ & $53.5 \pm 6.25$ & $54.71 \pm 4.27$ & $57.14 \pm 4.01$ & 0.081 \\
\hline $\operatorname{Sex}(M / F)$ & $11 / 9$ & $2 / 4$ & $2 / 5$ & $2 / 5$ & 0.45 \\
\hline Hemoglobin (gm/dL) & $13.52 \pm 1.93$ & $14.21 \pm 2.08$ & $14.32 \pm 1.81$ & $13.61 \pm 1.97$ & 0.74 \\
\hline PLT (cells × $10^{3} / \mathrm{uL}$ ) & $264.05 \pm 80.29$ & $239.5 \pm 71.57$ & $237.14 \pm 59.64$ & $92.85 \pm 16.03 \odot ® \infty$ & $0.001^{* *}$ \\
\hline WBCs (cells $\times 10^{3} / \mathrm{uL}$ ) & $6.62 \pm 2.41$ & $8.25 \pm 2.21$ & $7.41 \pm 2.25$ & $12.7 \pm 1.68 \odot ® \infty$ & $<0.001^{* *}$ \\
\hline Cholesterol (mg/dL) & $125.2 \pm 15.98$ & $189.5 \pm 7.17 \odot$ & $171.42 \pm 19.3 \odot \circledast$ & $188.57 \pm 11.07 \odot \infty$ & $<0.001^{* *}$ \\
\hline $\mathrm{TG}(\mathrm{mg} / \mathrm{dL})$ & $71.9 \pm 22.74$ & $136 \pm 12.08 \odot$ & $140.14 \pm 8.41 \odot$ & $144 \pm 4.86 \odot$ & $<0.001^{* *}$ \\
\hline $\mathrm{HDL}(\mathrm{mg} / \mathrm{dL})$ & $61 \pm 12.35$ & $38.66 \pm 7.03 \odot$ & $37.57 \pm 7.36 \odot$ & $32.14 \pm 5.66 \odot$ & $<0.001^{* *}$ \\
\hline LDL (mg/dL) & $83.6 \pm 25.14$ & $122.5 \pm 15.59 \odot$ & $129.85 \pm 11.18 \odot$ & $133.57 \pm 10.69 \odot$ & $<0.001^{* *}$ \\
\hline $\mathrm{RBS}(\mathrm{mg} / \mathrm{dL})$ & $117.1 \pm 17.56$ & $127.33 \pm 12.32$ & $124.57 \pm 13.4$ & $122.57 \pm 15.95$ & 0.477 \\
\hline Urea (mg/dL) & $11.65 \pm 5.03$ & $11.66 \pm 2.16$ & $14.14 \pm 3.89$ & $21.42 \pm 2.57 \bigcirc \oplus \infty$ & $<0.001^{* *}$ \\
\hline Blood Urea Nitrogen (mg/dL) & $6.05 \pm 2.12$ & $6.16 \pm 1.16$ & $7.14 \pm 1.95$ & $10.71 \pm 1.49 \odot \oplus \infty$ & $<0.001^{* *}$ \\
\hline Creatinine $(\mathrm{mg} / \mathrm{dL})$ & $0.83 \pm 0.18$ & $0.98 \pm 0.14$ & $0.9 \pm 0.19$ & $1.04 \pm 0.15$ & 0.054 \\
\hline CK-MB (ug/L) & $8.9 \pm 1.01$ & $3.83 \pm 1.28 \odot$ & $20.57 \pm 8.25 \odot \circledast$ & $187.42 \pm 11.16 \odot ® \infty$ & $<0.001^{* *}$ \\
\hline SIRT1 (ng/mL) & $5.99 \pm 6.23$ & $3.77 \pm 5.62 \odot$ & $1.01 \pm 1.58 \odot ®$ & $1.03 \pm 1.52131 \odot ®$ & $0.04^{*}$ \\
\hline
\end{tabular}

CCS: chronic coronary syndrome; UA: unstable angina; AMI: acute myocardial infarction; PLT: platelet count; WBCs: white blood cells; TG: triglycerides; HDL: highdensity lipoprotein; LDL: low-density lipoprotein; RBS: random blood sugar; CK-MB: creatine kinase-MB; SIRT1: sirtuin 1; $: p<0.05:$ statistically significant; **: $p<0.001$ : highly statistically significant; $\odot$ : significant difference with control; $®$ : significant difference with CCC; $\infty$ : significant difference with UA

significantly higher in the CAD groups. There was a significant difference in the plasma level of SIRT1 between the study groups $(p=0.04)$. The plasma SIRT1 level was significantly lower in patients with CCS than in patients in the control group, and was significantly lower in patients with either AMI or UA than in patients of the control and CCS groups $(p<0.05)$, with no significant difference found between patients with AMI and UA $(p>0.05)$ (Table 1).

There was a significant positive correlation between plasma SIRT1 and platelet count $(\mathrm{r}=0.333, p=0.036)$ and a significant negative correlation between plasma SIRT1 and both cholesterol $(\mathrm{r}=-0.367, p=0.02)$ and TG $(\mathrm{r}=-0.474, p=0.002)$ (Table 2).

There was a significant association and agreement between SIRT1 (at cutoff level $<2.4$ ) and CAD in general $(p=0.049)$ (Table 3). Regarding the receiver operating characteristics (ROC) curve, the plasma cutoff level of SIRT1 in CAD patients was $65.0 \%$ accurate in predicting CAD with a significant area under the curve $(p=0.013)$ at a cutoff value of less than $2.4 \mathrm{ng} / \mathrm{mL}$ with a sensitivity of $75.0 \%$, specificity of $55.0 \%$, positive predictive value (PPV) of $62.5 \%$, and negative predictive value (NPV) of 68.7\% (Tables 4, 5, Fig. 1).

The plasma level of SIRT1 in UA or AMI patients was $60.0 \%$ accurate in predicting UA or AMI with a significant area under the curve $(p=0.014)$ at a cutoff value of less than $0.66 \mathrm{ng} / \mathrm{mL}$ with a sensitivity of $64.3 \%$, specificity of $57.7 \%$, PPV of $45.5 \%$, and NPV of $75.0 \%$ (Tables 6 , 7, Fig. 2).

\section{Discussion}

CVD are still the leading cause of death worldwide. Inflammation plays a substantial role in the initiation and propagation of the atherosclerotic process [16]. Coronary artery luminal obstructions and plaque cracks due to atherosclerosis are the most frequent causes of CAD [17]. Apoptosis and necrosis of cardiomyocytes, endothelial cells, and monocytes with severe

Table 2 Correlations between Sirtuin1 and other studied parameters:

\begin{tabular}{lcl}
\hline & $r$ & $p$ \\
\hline Age (years) & -0.309 & 0.052 \\
Hemoglobin (g/dL) & -0.222 & 0.169 \\
WBCs $\left(10^{3} / \mathrm{cL}\right)$ & -0.093 & 0.569 \\
PLT $\left(10^{3} / \mathrm{uL}\right)$ & 0.333 & $0.036^{*}$ \\
RBS (mg/dL) & 0.073 & 0.656 \\
Urea (mg/dL) & -0.117 & 0.474 \\
Blood Urea Nitrogen (mg/dL) & -0.068 & 0.675 \\
Creatinine (mg/dL) & -0.152 & 0.348 \\
Cholesterol (mg/dL) & -0.367 & $0.02^{*}$ \\
TG (mg/dL) & -0.474 & $0.002^{*}$ \\
LDL (mg/dL) & -0.250 & 0.120 \\
HDL (mg/dL) & 0.287 & 0.073 \\
CK-MB (ug/L) & -0.282 & 0.077 \\
\hline
\end{tabular}

WBCs: white blood cells; PLT: platelet count; RBS: random blood sugar; TG: triglycerides; HDL: high-density lipoprotein; LDL: low-density lipoprotein; CK-MB: creatine kinase-MB

*: $p<0.05$ statistically significant 
Table 3 Association and agreement between Sirtuin 1 cutoff levels and coronary artery disease

\begin{tabular}{|c|c|c|c|c|c|c|}
\hline & \multicolumn{2}{|l|}{ Group } & \multirow[t]{2}{*}{ Total } & \multirow[t]{2}{*}{$x^{2}$} & \multirow[t]{2}{*}{$p$} & \multirow{2}{*}{$\begin{array}{l}\text { Kappa } \\
\text { agreemen }\end{array}$} \\
\hline & Control & CAD & & & & \\
\hline \multicolumn{7}{|c|}{ SIRT1 (ng/mL) cutoff } \\
\hline \multicolumn{7}{|c|}{$>2.4$} \\
\hline$N$ & 22 & 10 & 32 & 3.75 & $0.049^{*}$ & 0.32 \\
\hline$\%$ & $59.3 \%$ & $29.6 \%$ & $44.4 \%$ & & & \\
\hline \multicolumn{7}{|l|}{$<2.4$} \\
\hline $\mathrm{N}$ & 18 & 30 & 48 & & & \\
\hline$\%$ & $40.7 \%$ & $70.4 \%$ & $55.6 \%$ & & & \\
\hline \multicolumn{7}{|l|}{ Total } \\
\hline$N$ & 40 & 40 & 80 & & & \\
\hline$\%$ & $100.0 \%$ & $100.0 \%$ & $100.0 \%$ & & & \\
\hline
\end{tabular}

*: $p<0.05$ statistically significant

Table 4 Receiver operating characteristics (ROC) curve for prediction of coronary artery disease. Area under curve (AUC) of sirtuin 1 regarding coronary artery disease:

\begin{tabular}{lllll}
\hline $\begin{array}{l}\text { Area under } \\
\text { curve (AUC) }\end{array}$ & Cutoff & $\boldsymbol{p}$ & \multicolumn{2}{l}{$\mathbf{9 5 \% \text { Confidence interval }}$} \\
\cline { 3 - 5 } & & & Lower bound & Upper bound \\
\hline 0.729 & $<2.4$ & $0.013^{*}$ & 0.573 & 0.884 \\
\hline
\end{tabular}

*: $p<0.05$ statistically significant

Table 5 Validity of sirtuin 1 for prediction of coronary artery disease

\begin{tabular}{llllll}
\hline & Sensitivity & Specificity & $\begin{array}{l}+ \text { VE } \\
\text { predictive }\end{array}$ & $\begin{array}{l}\text {-VE } \\
\text { predictive }\end{array}$ & Accuracy \\
\hline $\begin{array}{l}\text { Sir- } \\
\text { tuin1 } \\
\text { cutoff }\end{array}$ & $75.0 \%$ & $55.0 \%$ & $62.5 \%$ & $68.7 \%$ & $65.0 \%$ \\
\hline
\end{tabular}

inflammation are the major causes of vessel damage under CAD. Thus, strategies that repress cell death and manage unsuitable proinflammatory responses are potential therapeutic strategies for improving the clinical prognosis of patients with CAD [18].

SIRTs are $\mathrm{NAD}^{+}$-dependent histone deacetylases that are implicated in a variety of cellular functions, including cell cycle regulation and cellular metabolism. Among the seven known human sirtuins, SIRT1 is implicated in a wide range of cellular functions [19].

Baur et al. [20] proposed a role of SIRT1 in aging and diseases that involve ischemia/reperfusion and neurodegeneration [20]. Hsu et al. [21] showed that cardiac-specific knockout SIRT1 mice exhibit a significant increment in the size of the risky myocardial infarction

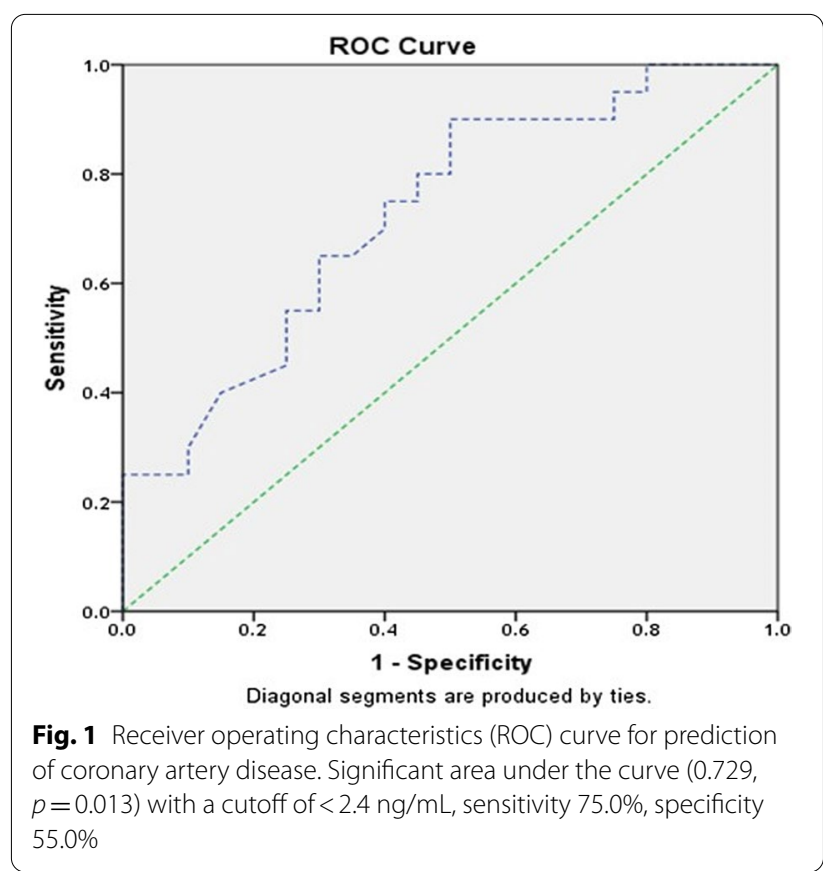

Table 6 Receiver operating characteristics (ROC) curve for sirtuin 1 cutoff level in prediction of unstable angina and AMI

\begin{tabular}{|c|c|c|c|}
\hline \multirow{2}{*}{$\begin{array}{l}\text { Area under curve } \\
\text { (AUC) }\end{array}$} & \multirow[t]{2}{*}{ Cutoff $p$} & \multicolumn{2}{|c|}{ 95\% Confidence interval } \\
\hline & & Lower bound & Upper bound \\
\hline 0.738 & $<0.660 .014^{*}$ & 0.583 & 0.892 \\
\hline
\end{tabular}

*:p<0.05: statistically significant

area [21]. The authors concluded that SIRT1 has a cardioprotective effect. SIRT1 induces the upregulation of cardioprotective molecules and downregulation of proapoptotic molecules, thereby attenuating oxidative stress, 
Table 7 Validity of sirtuin 1 in prediction of patients with AMI or unstable angina

\begin{tabular}{llllll}
\hline & Sensitivity & Specificity & PPV & NPV & Accuracy \\
\hline $\begin{array}{l}\text { Sirtuin1 level } \\
\text { cutoff }\end{array}$ & $64.3 \%$ & $57.7 \%$ & $45.0 \%$ & $75.0 \%$ & $60.0 \%$ \\
\hline
\end{tabular}

PPV: positive predictive value; NPV: negative predictive value

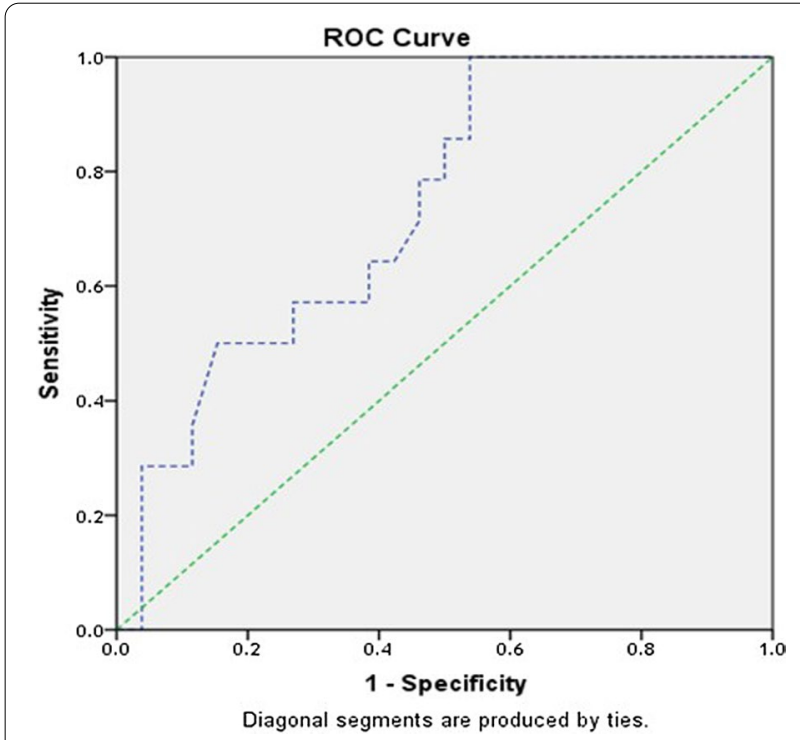

Fig. 2 Receiver operating characteristics (ROC) curve for prediction of unstable angina or acute myocardial infarction. Significant area under the curve $(0.738, p=0.014)$ with cutoff of $<0.66 \mathrm{ng} / \mathrm{mL}$, sensitivity $64.3 \%$, specificity $57.7 \%$

and inhibiting apoptosis. Consequently, the activation of SIRT1 could be a novel method of cardioprotection.

In addition, SIRT1 inhibition causes oxidative stress and inflammation in patients with CAD, whereas the activation of SIRT1 function reverses these atherosclerotic events which may provide new knowledge that is relevant for the management of CAD patients [9].

Stein and Matter [22] demonstrated that SIRT1 delays the progression of atherosclerosis by preventing macrophage foam cell formation [22].

Breitenstein et al. [23] also found that the SIRT1 expression in monocytes was lower in ACS patients. SIRT1 exerts atheroprotective effects on the vascularity by downregulating the expression of various proinflammatory cytokines and mediating vasodilatation via the actions of eNOS-derived nitric oxide and scavenging reactive oxygen species [23].

To our knowledge, few studies have assessed the serum level of SIRT1 in patients with CAD $[11,12]$.
The present study aimed to measure the plasma levels of SIRT1 in CAD patients and to explore its relationship with cardiovascular risk factors.

Our results showed that plasma SIRT1 levels were significantly lower in the UA and AMI groups than in the CCS and control groups. However, the difference between the AMI and CCS groups was not statistically significant, while the plasma SIRT1 level was significantly lower in the CCS group than in the control group. Similar to our results, Doulamis et al. [11] reported a significantly low level of serum SIRT1 in patients with advanced CAD. Moreover, they noticed an increased prevalence of AMI in patients with low SIRT1 levels [11].

Another study by Mariani et al. [13] found that circulating SIRT1 is inversely correlated with epicardial fat thickness, which is a candidate marker of cardiac ischemia. The authors suggested that plasma SIRT1 measurement might provide additional information for risk assessment of CAD, especially in obese people [13]. Low plasma SIRT1 levels in CAD patients may be due to its consumption in preventing the hazards of cardiac ischemia.

Another study reported that the cardiomyocyte-specific deletion of the SIRT1 gene sensitizes the myocardium to ischemia and reperfusion injury [14], which indicates that changes in SIRT1 may be a cause of cardiac ischemia.

Fry et al. [24] assessed the SIRT1 level in media other than plasma or serum in CAD patients. The authors found that vascular smooth muscle SIRT1 protects against aortic stiffness, which is a major risk factor for IHD [24].

Several studies have also reported that SIRT1 expression is reduced in the monocytes of patients with CAD, that the SIRT1 gene plays a protective role against ACS and that the activation of SIRT1 function reverses atherosclerotic events $[9,23,25]$.

Interestingly, Li et al. [26] reported that SIRT1 expression significantly correlates with inflammatory cytokine levels in patients with CAD but not with the severity of coronary lesions [26].

Yamac and Kilic [27] noticed a significant increase in SIRT1 levels and expression in CAD patients who received statin therapy and concluded that SIRT1 might have a cardioprotective role after AMI. However, Kilic et al. [28] previously reported (in 2015) that the protective effect of statin treatment on CVD is through the inhibition of SIRT1 expression.

The present study showed no significant correlation between SIRT1 with age. This can be explained by the limited age range of the participants selected. However, 
few studies have assessed the negative correlation between SIRT1 and age [30, 31]. Engelfriet et al. [29] found that the SIRT1 level in blood lymphocytes might be a promising biochemical marker associated with aging [19].

In our study, there was a significant difference between serum urea and BUN levels between the study groups as they were significantly higher in the AMI group than in the other groups. In agreement with our findings, a prospective study carried out by Horiuchi et al. in 2018 found that BUN and serum urea levels were significantly higher in ACS patients and can be useful predictors of ACS [32].

We did not notice, however, a significant correlation between plasma SIRT1 levels and blood urea levels. This result is contrary to that obtained by Doulamis et al. [11], who reported a significant negative correlation [11].

In our study, serum total cholesterol, TG, and LDL were significantly lower, and HDL was significantly higher in the control group than in the CAD groups. In agreement with our results, Dobiasova and Frohlich [33] observed an inverse correlation between baseline HDL levels and both cardiovascular and all-cause death in the general population. Moreover, they found a significant inverse correlation of HDL with LDL particles, which are strongly correlated with the initiation and progression of atherosclerosis [33]. Moreover, Mendivil et al. [34] associated the risk contributed by LDL to the presence of apolipoprotein C-III [34].

In our study, there was a significant difference in CK-MB levels between the study groups. These levels were higher in the CAD group. Our results agree with those of Chan et al. [9], who found that creatine kinase, total cholesterol, and LDL concentrations were higher in CAD patients than in control subjects [9].

In our study, a significant positive correlation was found between plasma SIRT1 levels and platelet counts. This is similar to the results of Moscardó et al. [35]. The authors demonstrated that the inhibition of SIRT1 was associated with a concentration-dependent inhibition of the platelet responses, including platelet aggregation, dense granule secretion, and increase in cytosolic calcium levels, suggesting a regulatory role for SIRT1 in platelet responses.

In the present study, the plasma SIRT1 level was also found to be significantly negatively correlated with both cholesterol and TG. These results are congruent with those of Li et al. [36], who demonstrated that SIRT1 activates liver X receptors (LXRs), which in turn regulate the transfer of cholesterol from peripheral tissues to the liver (reverse cholesterol transport), thereby regulating cholesterol homeostasis [36].
Differences between our results and those of other studies may be explained by differences in ethnicity, lifestyle, and characteristics of the patients included.

\section{Conclusions}

Our results demonstrated that the plasma levels of SIRT1 were lower in CAD patients compared to normal control. Besides, there was a significant positive correlation between plasma SIRT1 and platelet count and a significant negative correlation between plasma SIRT1 and both cholesterol and TG. We predicted that low SIRT1 could be a new risk factor for CAD and SIRT1 activation could be a possible preventive factor of CAD. However, this needs to be validated by further molecular studies with multi-center cooperation, different ethnic groups, and follow-up measurements.

\section{Abbreviations \\ CAD: Coronary artery disease; SIRT1: Silent information regulator 1; CCS: Chronic coronary syndrome; AMI: Acute myocardial infarction; UA: Unstable angina; NAD: Nicotinamide adenine dinucleotide; CBC: Complete blood count; RBS: Random blood sugar; CK-MB: Creatine kinase-MB; PPV: Positive predictive value; NPV: Negative predictive value; CVD: Cardiovascular diseases; ROS: Reactive oxygen species.}

\section{Acknowledgements \\ Not applicable.}

\section{Authors' contributions}

IE and AMA designed and directed the project. RMA, AAA, and SH performed the experiments and analyzed the data. AAA and SH wrote the manuscript. All authors have read and approved the manuscript.

\section{Funding}

Not applicable.

\section{Availability of data and materials}

The data that support the findings of this study are available from the corresponding author upon reasonable request.

\section{Declarations}

Ethics approval and consent to participate

Written informed consent was obtained from the patients or their relatives with an explanation of the study procedure and possible associated hazards. The study was approved by Institution Review Board (IRB), Faculty of Medicine, Zagazig University (ZU-IRB\# 3931/13--2017).

\section{Consent for publication}

Not applicable.

\section{Competing interests}

The authors have no conflict of interest to declare.

\section{Author details}

${ }^{1}$ Internal Medicine Department, Faculty of Medicine, Zagazig University, Zagazig, Egypt. ${ }^{2}$ Medical Biochemistry and Molecular Biology Department, Faculty of Medicine, Zagazig University, Zagazig, Egypt.

Received: 12 August 2021 Accepted: 12 October 2021

Published online: 04 November 2021 


\section{References}

1. Than M, Cullen L, Reid CM, Lim SH, Aldous S, Ardagh MW, PeacockWF, Parsonage WA, Ho HF, Ko HF, Kasliwal RR, Bansal M, Soerianata S, Hu D, Ding R, Hua Q, Seok-Min K, Sritara P, Sae-Lee R, Chiu TF, Tsai KC, Chu FY, Chen WK, Chang WH, Flaws DF, George PM, Richards AM (2011) A 2-h diagnostic protocol to assess patients with chest pain symptoms in the Asia-Pacific region (ASPECT): a prospective observational validation study. Lancet 377:1077-1084

2. Libby P, Theroux P (2005) Pathophysiology of coronary artery disease. Circulation 111:3481-3488

3. Morris KC, Lin HW, Thompson JW, Perez-Pinzon MA (2011) Pathways for ischemic cytoprotection: role of sirtuins in caloric restriction, resveratrol, and ischemic preconditioning. J Cereb Blood Flow Metab 31:1003-1019

4. Hwang JW, Yao H, Caito S, Sundar IK, Rahman I (2013) Redox regulation of SIRT1 in inflammation and cellular senescence. Free Radic Biol Med 61:95-110

5. Matsushima S, Sadoshima J (2015) The role of sirtuins in cardiac disease. Am J Physiol Heart Circ Physiol 309:H1375-H1389

6. Rutanen J, Yaluri N, Modi S, Pihlajamäki J, Vänttinen M, Itkonen P, Kainulainen S, Yamamoto H, Lagouge M, Sinclair DA, Elliott P, Westphal C, Auwerx J, Laakso M (2010) SIRT1 mRNA expression may be associated with energy expenditure and insulin sensitivity. Diabetes 59:829-835

7. Ianni A, Yuan X, Bober E, Braun T (2018) Sirtuins in the cardiovascular system: potential targets in pediatric cardiology. Pediatr Cardiol 39:983-992

8. Zullo A, Simone E, Grimaldi M, Musto V, Mancini FP (2018) Sirtuins as mediator of the anti-ageing effects of calorie restriction in skeletal and cardiac muscle. Int J Mol Sci 19:928

9. Chan SH, Hung CH, Shih JY, Chu PM, Cheng YH, Lin HC, Tsai KL (2017) SIRT1 inhibition causes oxidative stress and inflammation in patients with coronary artery disease. Redox Biol 13:301-309

10. Bindu S, Pillai VB, Gupta MP (2016) Role of sirtuins in regulating pathophysiology of the heart. Trends Endocrinol Metab 27:563-573

11. Doulamis IP, Tzani Al, Konstantopoulos PS, Samanidis G, Georgiopoulos G, Toutouzas KP, Perrea DN, Perreas KG (2017) A sirtuin 1/MMP2 prognostic index for myocardial infarction in patients with advanced coronary artery disease. Int J Cardiol 230:447-453

12. Kızltunç E, Kösem A, Özkan C, Ilgın BU, Kundi H, Çetin M, Ornek E (2019) Serum sirtuin 1, 3 and 6 levels in acute myocardial infarction patients. Arq Bras Cardiol 113(1):33-39

13. Mariani S, Costantini D, Lubrano C, Basciani S, Caldaroni C, Barbaro G, Poggiogalle E, Donini LM, Lenzi A, Gnessi L (2016) Circulating SIRT1 inversely correlates with epicardial fat thickness in patients with obesity. Nutr Metab Cardiovasc Dis 26:1033-1038

14. Wang L, Quan N, Sun W, Chen X, Cates C, Rousselle T, Zhou X, Zhao X, Li J (2018) Cardiomyocyte-specific deletion of Sirt1 gene sensitizes myocardium to ischaemia and reperfusion injury. Cardiovasc Res 114:805-82

15. Mericskay M (2016) Nicotinamide adenine dinucleotide homeostasis and signalling in heart disease: pathophysiological implications and therapeutic potential. Arch Cardiovasc Dis 109:207-215

16. Roth GA, Forouzanfar MH, Moran AE, Barber R, Nguyen G, Feigin VL, Naghavi M, Mensah GA, Murray CJ (2015) Demographic and epidemiologic drivers of global cardiovascular mortality. N Engl J Med 372:1333-1341

17. Yahagi K, Kolodgie FD, Lutter C, Mori H, Romero ME, Finn AV, Virmani R (2017) Pathology of human coronary and carotid artery atherosclerosis and vascular calcification in diabetes mellitus. Arterioscler Thromb Vasc Biol 37:191-204

18. Orogo AM, Gustafsson AB (2013) Cell death in the myocardium: my heart will not go on. IUBMB Life 65:651-656

19. Bassett SA, Barnett MP (2014) The role of dietary histone deacetylases (HDACs) inhibitors in health and disease. Nutrients 6:4273-4301

20. Baur JA, Ungvari Z, Minor RK, Le Couteur DG, de Cabo R (2012) Are sirtuins viable targets for improving healthspan and lifespan? Nat Rev Drug Discov 11:443-461
21. Hsu CP, Zhai P, Yamamoto T, Maejima Y, Matsushima S, Hariharan N, Shao D, Takagi H, Oka S, Sadoshima J (2010) Silent information regulator 1 protects the heart from ischemia/reperfusion. Circulation 122:2170-2182

22. Stein S, Matter CM (2011) Protective roles of SIRT1 in atherosclerosis. Cell Cycle 10:640-647

23. Breitenstein A, Wyss CA, Spescha RD, Franzeck FC, Hof D, Riwanto M, Hasun M, Akhmedov A, von Eckardstein A, Maier W, Landmesser U, Lüscher TF, Camici GG (2013) Peripheral blood monocyte Sirt1 expression is reduced in patients with coronary artery disease. PLoS ONE 8:e53106

24. Fry JL, Al Sayah L, Weisbrod RM, Van Roy I, Weng X, Cohen RA, Bachschmid MM, Seta F (2016) Vascular smooth muscle Sirtuin-1 protects against diet-induced aortic stiffness. Hypertension 68:775-784

25. Hu Y, Wang L, Chen S, Liu X, Li H, Lu X, Yang X, Huang J, Gu D (2015) Association between the SIRT1 mRNA expression and acute coronary syndrome. J Atheroscler Thromb 22:165-182

26. Li Y, Ni J, Guo R, Li W (2016) In patients with coronary artery disease and Type 2 Diabetes, SIRT1 expression in circulating mononuclear cells is associated with levels of inflammatory cytokines but not with coronary lesions. Biomed Res Int 2016:8734827

27. Yamaç AH, Kılıç Ü (2018) Effect of statins on sirtuin 1 and endothelial nitric oxide synthase expression in young patients with a history of premature myocardial infarction. Turk Kardiyol Dern Ars 46:205-215

28. Kilic U, Gok O, Elibol-Can B, Uysal O, Bacaksiz A (2015) Efficacy of statins on sirtuin 1 and endothelial nitric oxide synthase expression: the role of sirtuin 1 gene variants in human coronary atherosclerosis. Clin Exp Pharmacol Physiol 42:321-330

29. Engelfriet PM, Jansen EH, Picavet HS, Dollé ME (2013) Biochemical markers of aging for longitudinal studies in humans. Epidemiol Rev 35:132-151

30. Kumar R, Chaterjee P, Sharma PK, Singh AK, Gupta A, Gill K, Tripathi M, Dey AB, Dey S (2013) Sirtuin1, a promising serum protein marker for early detection of Alzheimer's disease. PLoS ONE 8:e61560

31. Xia X, Chen W, McDermott J, Han JJ (2017) Molecular and phenotypic biomarkers of aging. F1000Res 6:860

32. Horiuchi Y, Aoki J, Tanabe K, Nakao K, Ozaki Y, Kimura K, Ako J, Yasuda S, Noguchi T, Suwa S, Fujimoto K, Nakama Y, Morita T, Shimizu W, Saito Y, Hirohata A, Morita Y, Inoue T, Okamura A, Uematsu M, Hirata K, Shibata Y, Nakai M, Nishimura K, Miyamoto Y, Ishihara M, J-MINUET investigators (2018) A high level of blood urea nitrogen is a significant predictor for inhospital mortality in patients with acute myocardial infarction. Int Heart J 59:263-271

33. Dobiásová M, Frohlich J (2001) The plasma parameter log (TG/HDL-C) as an atherogenic index: correlation with lipoprotein particle size and esterification rate in apoB-lipoprotein-depleted plasma (FER (HDL)). Clin Biochem 34:583-588

34. Mendivil CO, Rimm EB, Furtado J, Chiuve SE, Sacks FM (2011) Low-density lipoproteins containing Apolipoprotein C-III and the risk of coronary heart disease. Circulation 124:2065-2072

35. Moscardó A, Vallés J, Latorre A, Jover R, Santos MT (2015) The histone deacetylase sirtuin 2 is a new player in the regulation of platelet function. J Thromb Haemost 13:1335-1344

36. Li X, Zhang S, Blander G, Tse JG, Krieger M, Guarente L (2007) SIRT1 deacetylates and positively regulates the nuclear receptor LXR. Mol Cell 28:91-106

\section{Publisher's Note}

Springer Nature remains neutral with regard to jurisdictional claims in published maps and institutional affiliations. 